\title{
Control Techniques for a Class of Fractional Order Systems
}

\author{
Mircea Ivanescu ${ }^{1, *}$, Ioan Dumitrache ${ }^{2}$, Nirvana Popescu ${ }^{3}$ and Decebal Popescu ${ }^{3}$ \\ 1 Department of Mechatronics, University of Craiova, 200585 Craiova, Romania \\ 2 Department of Automation, University Politehnica of Bucharest, 060042 București, Romania; \\ ioandumitrache87@yahoo.com \\ 3 Department of Computer Science, University Politehnica of Bucharest, 060042 București, Romania; \\ nirvana.popescu@upb.ro (N.P.); decebal.popescu@upb.ro (D.P.) \\ * Correspondence: mirceaivanescu19@gmail.com or ivanescu@robotics.ucv.ro
}

check for updates

Citation: Ivanescu, M.; Dumitrache, I.; Popescu, N.; Popescu, D. Control Techniques for a Class of Fractional Order Systems. Mathematics 2021, 9, 2357. https://doi.org/10.3390/ math9192357

Academic Editors: Somayeh Mashayekhi and William S. Oates

Received: 3 August 2021

Accepted: 18 September 2021

Published: 23 September 2021

Publisher's Note: MDPI stays neutral with regard to jurisdictional claims in published maps and institutional affiliations.

\begin{abstract}
The paper discusses several control techniques for a class of systems described by fractional order equations. The paper presents the unit frequency criteria that ensure the closed loop control for: Fractional Order Linear Systems, Fractional Order Linear Systems with nonlinear components, Time Delay Fractional Order Linear Systems, Time Delay Fractional Order Linear Systems with nonlinear components. The stability criterion is proposed for the systems composed of fractional order subsystems. These techniques are used in two applications: Soft Exoskeleton Glove Control, studied as a nonlinear model with time delay and Disabled Man-Wheelchair model, analysed as a fractional-order multi-system.
\end{abstract}

Keywords: stability; frequency criterion; closed loop control; exoskeleton glove model; disabled man model

\section{Introduction}

In the last decade, the extension of the applications of the fractional order systems (FOS) has determined a corresponding increase of the control techniques, of the methods of investigation of the special requirements imposed by these systems. Enumerating these methods would be an extremely difficult operation. A summary of these efforts can be found in $[1,2]$. Seeking to capture certain aspects in this domain, we could identify some directions that mark this activity. The stability criteria of FOS are obtained both by conventional methods deriving from the study of transfer functions with fractional exponent [3-5] and from those derived from Lyapunov's second method [6-10]. Mittag-Leffler stability is studied by Lyapunov techniques in [11]. FOS models defined by Caputo derivatives are analyzed in $[12,13]$. Nonlinear FOS or linear FOS with nonlinear components are treated in [14-19]. A physical interpretation of fractional viscoelasticity based on the fractal structure of media is investigated in [20]. Nonlinear optimal control techniques are proposed in [21]. Other control and stability issues refer to delayed FOS models. Stability criteria are formulated in the form of algebraic criteria [22-24] or are derived by analytical techniques derived from the Lyapunov method [25-28]. The control of multi-system architectures defined by FOS models is discussed in [29-31]. Frequency criteria for the control of Linear FOS are presented in [32-34]. A comprehensive review of literature related to the industrial use and integration of FOPID control is presented in [35]. FPGA implementation techniques of Fractional Order Systems is discussed in [36] and analog-hardware implementation of a FOPID for a DC motor [37] is emphasized. Memristor-based implementation with neuromorphic circuits which provide guidelines for physical realization of FOPID controllers, and a discrete implementation are presented in [38,39] respectively. Motivated by the above discussion, our main objective in this paper is to develop new stability criteria for FOS models via the approach in which the control laws are defined by frequency criteria. The main contribution of this paper, compared with the existing results, are listed as follows: 
- Frequency criterion for linear FOS is proposed. Lyapunov techniques and the methods that derive from Yakubovici-Kalman-Popov Lemma [40] are used and the criteria that ensure asymptotic stability of the closed loop system are inferred. The criterion is extended to linear FOS with nonlinear components.

- Frequency criterion for time delay FOS is proposed using an approximate model based on Grunwald-Letnikov formula. The result is extended to the systems with nonlinear components.

- Stability criterion is proposed for systems composed of fractional order subsystems.

- The proposed methods have been implemented on two major applications: Soft Exoskeleton Glove Control that is studied as a nonlinear FOS model with time delay and Disabled Man-Wheelchair system that is studied as a fractional-order multi- system.

The paper is structured as follows: Section 2 presents mathematical preliminaries, and the control techniques proposed in this paper, Section 3 treats two applications: Soft Exoskeleton Glove Control and Disabled Man-Wheelchair system control, Section 4 is dedicated to Discussion and Section 5 presents Conclusions.

\section{Control Techniques}

Notations 1. Let $X \in R^{n \times n}$ be a real matrix. We denote the eigenvalues of $X$ by $\lambda_{i}(X)$. We denote by $\lambda_{\min (X)}, \lambda_{\max (X)}$, the minimum eigenvalue and maximum eigenvalue of $X$, respectively. Write $X \succcurlyeq(\succ) 0$, if $X$ is a positive semidefinite (positive definite) matrix. $X \succcurlyeq Y$ if $X-Y \succcurlyeq 0$. The identity matrix in $R^{n \times n}$ is shown by $I$. Let $z \in R^{n}$ be a vector, $\|z\|=\left(\sum z_{i}^{2}\right)^{\frac{1}{2}}$. We denote by $j$ the complex number, $j^{2}=-1$.

\subsection{Mathematical Preliminaries}

Lemma 1. Ref. [29] For matrix $P \in R^{n x n}, P=P^{T} \succ 0$, the following inequality holds:

$$
\lambda_{\min (P)} \mathrm{I} \preccurlyeq P \preccurlyeq \lambda_{\max (P)} \mathrm{I}
$$

Consider the fractional order system

$$
D^{\beta} z(t)=A z(t)
$$

where $D^{\beta} z(t)$ is the Caputo derivative of $z(t)$ for $0<\beta<1$.

Definition 1. The matrix $A$ is Hurwitz stable if

$$
\operatorname{Arg}(\operatorname{eig}(A))>\frac{\pi}{2}
$$

Definition 2. The matrix $A$ is $\beta$-fractional order stable if

$$
\operatorname{Arg}(\operatorname{eig}(A))>\beta \frac{\pi}{2}
$$

Theorem 1. Refs. $[6,14,15]$ The system (1), where $A$ is $\beta$-fractional order stable, is asymptotically stable.

Theorem 2. Refs. $[6,9,14,15]$ The system $D^{\beta} z(t)=f(z(t)), z\left(t_{0}\right)=z_{0}$ is asymptotically stable if there exist a continuously differentiable function $V(t, z)$ that satisfies

$$
\alpha_{1}\|z\|^{2} \leq V(t, z(t)) \leq \alpha_{2}\|z\|^{2}
$$




$$
D^{\beta} V(t, z(t)) \leq-\alpha_{3}\|z\|^{2}
$$

where $\alpha_{1}, \alpha_{2}, \alpha_{3}$ are positive constants, $0<\beta<1$.

Lemma 2. Refs. $[16,18]$ Let $z(.) \in R^{n}$ be a differentiable vector function. Then, the following inequality holds:

$$
\frac{1}{2} D^{\beta}\left[z^{T}(t) z(t)\right] \leq z^{T}(t) D^{\beta} z(t), \beta \in(0,1), t \geq t_{0}
$$

\subsection{Control Algorithms}

2.2.1. Fractional Order Linear Systems (FOLS)

Consider the following FOLS model

$$
\begin{gathered}
D^{\beta} z(t)=A z(t)+b u(t) \\
z\left(t_{0}\right)=z_{0} \\
y(t)=c^{T} z(t)
\end{gathered}
$$

where $D^{\beta} z(t)$ is the Caputo derivative of $z(t)$ for $0<\beta<1, A, b, c$ are $(n \times n),(n \times 1),(n \times 1)$ respectively, constant matrices, $z \in S \complement R^{n}, y \in R^{1}, u \in R^{1}$ are the system state, output and input.

Consider the following control law

$$
u(t)=-k y(t)
$$

where the controller gain $k=k_{1}+k_{2}$ is a positive constant that verifies the conditions

$$
\begin{gathered}
\operatorname{Arg}\left(\operatorname{eig}\left(\left(A-k_{1} b c^{T}\right)\right)>\frac{\pi}{2}\right. \\
k_{2} \sigma \leq 1
\end{gathered}
$$

and $\sigma$ is a positive constant.

Theorem 3. The system (7)-(12) which satisfies the following condition

$$
\operatorname{Re}\left(c^{T}\left(j \omega \mathrm{I}-\left(A-k_{1} b c^{T}\right)\right)^{-1} b\right) \geq-\sqrt{\sigma},, \forall \omega \in(-\infty,+\infty)
$$

is asymptotically stable.

Proof. The following Lyapunov function will be considered

$$
V(z)=z^{T} P z
$$

where $P=P^{T} \succ 0$. This function verifies the conditions of Theorem 2 for $\alpha_{1}=\lambda_{\min (P)}$, $\alpha_{2}=\lambda_{\max (P)}[7,15]$.

$$
D^{\beta} V(z) \leq\left(D^{\beta} z^{T}\right) P z+z^{T} P\left(D^{\beta} z\right)
$$

By evaluating (15) along the solutions of (7)-(10), yields

$$
D^{\beta} V(z) \leq z^{T}\left(\left(A-k_{1} b c^{T}\right)^{T} P+P\left(A-k_{1} b c^{T}\right)\right) z+2 z^{T} P b u_{2}
$$

where $u_{2}=-k_{2} y$. This inequality can be rewritten as

$$
D^{\beta} V(z) \leq z^{T}\left(\left(A-k_{1} b c^{T}\right)^{T} P+P\left(A-k_{1} b c^{T}\right)\right) z+2 z^{T} P b u_{2}-z^{T} c u_{2}+y u_{2}
$$


Then by (10) and (12) it follows that

$$
y u_{2}<-\sigma u_{2}^{2}
$$

Substituting this result into (17) results in

$$
D^{\beta} V(z) \leq z^{T}\left(\left(A-k_{1} b c^{T}\right)^{T} P+P\left(A-k_{1} b c^{T}\right)\right) z+2 z^{T}\left(P b-\frac{1}{2} c\right) u_{2}-\sigma u_{2}{ }^{2}
$$

By employing Yakubovici-Kalman-Popov (YKP) Lemma [40] and the condition (11), it follows that

$$
\begin{gathered}
z^{T}\left(\left(A-k_{1} b c^{T}\right)^{T} P+P\left(A-k_{1} b c^{T}\right)\right) z=-q q^{T} \\
P b-\frac{1}{2} c=\sqrt{\sigma} q
\end{gathered}
$$

Now, substituting this result into (19) and considering the control law (10), it yields

$$
D^{\beta} V(z) \leq-z^{T}\left(q+k_{2} \sqrt{\sigma} c\right)\left(q+k_{2} \sqrt{\sigma} c\right)^{T} z
$$

or,

$$
D^{\beta} V(z) \leq-\rho\|z\|^{2}
$$

where

$$
\rho=\left\|\left(q+k_{2} \sqrt{\sigma} c\right)\left(q+k_{2} \sqrt{\sigma} c\right)^{T}\right\|
$$

2.2.2. Fractional Order Linear Systems with Nonlinear Components

Consider the following system

$$
D^{\beta} z(t)=A z(t)+b u(t)+f(z(t))
$$

where $A, b, c$ are defined as in (7), (9) and $f(z)$ is a nonlinear function $\left(f: R^{n} \rightarrow R^{n}, f(0)=0\right)$ that satisfies the condition

$$
\left|z^{T} f(z)\right| \leq \mu\|z\|^{2}, \mu>0
$$

Consider the control law (10)-(12).

Theorem 4. The system (25), (9) with the control law (10)-(12), which satisfies the following condition

$$
\operatorname{Re}\left(c^{T}\left(j \omega I-A^{*}\right)^{-1} b\right) \geq-\sqrt{\sigma},, \forall \omega \in(-\infty,+\infty)
$$

is asymptotically stable, where

$$
A^{*}=A-k_{1} b c^{T}+\mu \zeta(Q) \mathrm{I}
$$

is a Hurwitz matrix and $P$ is the solution of the Lyapunov equation, $A^{*} P+P A^{*}=-q q^{T}=-Q$ and $\|P\|<\lambda_{\max (P)}<\zeta(Q), \zeta$ represents the upper matrix bound of $P$ [29].

Proof. The proof is similar to that for Theorem 1 .

2.2.3. Time Delay Fractional Order Linear Systems (TDFOLS)

Consider the following TDFLOS model:

$$
D^{\beta} z(t)=A z(t)+A_{1} z(t-\tau)+b u(t), \mathrm{t} \in[0, \mathrm{~T}], \tau \in \Im
$$




$$
\begin{gathered}
z(t)=\varphi(t), t \in[-\tau, 0] \\
y(t)=c^{T} z(t),
\end{gathered}
$$

where $0<\beta<1, A, A_{1}, b, c$ are $(n \times n),(n \times n),(n \times 1),(n \times 1)$ respectively, constant matrices, $z \in R^{n}, y \in R^{1}, u \in R^{1}$ are the system state, output, and input.

Consider the control law

$$
u(t)=-k y(t)
$$

Assumption 1. The following constraint is satisfied:

$$
\left|z^{T}(t-\tau) z(t)\right| \leq \alpha(\tau) z^{T}(t) z(t), \tau \in \Im
$$

where $\Im=\left[\tau_{\min }, \tau_{\max }\right]$ and $\alpha(\tau)$ can be approximated by a linear relation

$$
\alpha(\tau)=\alpha_{1}+\alpha_{2} \tau^{\beta}, \tau \in \Im
$$

or,

$$
\left|z^{T}(t-\tau)\|z(t)\|\right| \leq \alpha_{\max } z(t)
$$

Assumption 2. There exist the control gain $k_{1}>0,\left(k_{1}<k\right)$ such that

$$
A^{*}=A-k_{1} b c^{T}, \tau \in \Im
$$

is a Hurwitz matrix. As in the previous sections, consider that $k_{2}=k-k_{1}$ verifies the condition

$$
k_{2} \sigma \leq 1
$$

Theorem 5. The system (29)-(31), with the control law (32) and which satisfies the following condition

$$
\begin{aligned}
\left.\operatorname{Re}\left(c^{T}\left(j \omega I-A^{*}\right)\right)^{-1} b\right) & \geq-\sqrt{\sigma}, \forall \omega \in(-\infty,+\infty) \\
\rho-\rho^{*} & >0
\end{aligned}
$$

is asymptotically stable, where $\rho, \rho^{*}$ are defined by (26), (42), respectively.

Proof. The Lyapunov function (16) is considered and, using the same techniques as in Section 2.2.1, taking into account the control law (32), the conditions (36), (37), and YKP Lemma (22), (23), it is obtained

$$
D^{\beta} V(z(t)) \leq-z(t)^{T}\left(q+k_{2} \sqrt{\sigma} c\right)\left(q+k_{2} \sqrt{\sigma} c\right)^{T} z(t)+z(t)^{T}\left(A_{1}^{T} P+P A_{1}\right) z(t-\tau)
$$

Therefore, applying inequalities (33), (35) yields

$$
D^{\beta} V(z(t)) \leq-z(t)^{T}\left(q+k_{2} \sqrt{\sigma} c\right)\left(q+k_{2} \sqrt{\sigma} c\right)^{T} z(t)+\rho^{*}\|z(t)\|^{2}
$$

where

$$
\rho^{*}=\lambda_{\max \left(A_{1}^{T} P+P A_{1}\right)} \alpha_{\max }
$$

The inequality (41) can be rewritten as

$$
D^{\beta} V(z(t)) \leq-\left(\rho-\rho^{*}\right)\|z(t)\|^{2}
$$

where $\rho$ is given by (26). 
Remark 1. The result of Theorem 3 can be easily extended to systems with nonlinear components,

$$
D^{\beta} z(t)=A z(t)+A_{1} z(t-\tau)+f(z)+b u(t), \mathrm{t} \in[0, \mathrm{~T}], \tau \in \Im
$$

where $f(z)$ verifies the condition (26). In this case, the frequential condition (38) is verified for $A^{*}+\mu \zeta(Q) I$, where $\mu, \zeta, Q$ are defined in Section 2.2.2.

\subsubsection{Systems Composed of Linear Fractional Order Subsystems}

Consider the linear systems

$$
\begin{gathered}
D^{\beta} z^{i}(t)=A_{i} z^{i}(t), i=1,2, \ldots, N \\
z^{i}\left(t_{0}\right)=z_{0}^{i}
\end{gathered}
$$

where the exponent, $A_{i}$ is a $\left(n_{i} \times n_{i}\right)$ constant matrix, $z^{i} \in S^{i} \complement R^{n_{i}}$ In the composite system, a number of components can be $\beta$-fractional order stable systems,

$$
\operatorname{Arg}\left(\operatorname{eig}\left(A_{j}\right)\right)>\beta \frac{\pi}{2}, j=1,2, \ldots, K, K \leq N
$$

Consider the Lyapunov function associated with each system $i,\left(V_{i}: R^{n_{i}} \rightarrow R^{1}\right)$,

$$
V_{i}\left(z^{i}(t)\right)=z^{i T}(t) z^{i}(t)
$$

From (4) and Lemma 1 (6), yields

$$
D^{\beta} V_{i}\left(z^{i}(t)\right) \leq 2 z^{i T}(t) D^{\beta}\left(z^{i}(t)\right)=2 z^{i T}(t) A_{i} z^{i}(t), i=1,2, \ldots, N
$$

or

$$
D^{\beta} V_{i}\left(z^{i}(t)\right) \leq 2 \lambda_{\max \left(A_{i}\right)} z^{i T}(t) z^{i}(t)=2 \lambda_{\max \left(A_{i}\right)} V_{i}\left(z^{i}(t)\right)
$$

Consider the following composite system

$$
D^{\beta} z^{i}(t)=A_{i} z^{i}(t)+\sum_{j=1}^{N} B_{i j} z^{j}(t), i=1,2, \ldots, N
$$

where $B_{i j}$ is a $\left(n_{i} \times n_{j}\right)$ inter-connection matrix that satisfies the following condition

$$
z^{i T}(t) \sum_{j=1}^{N} B_{i j} z^{j}(t) \leq \sum_{j=1}^{N} \gamma_{i j} z^{j T}(t) z^{j}(t), i=1,2, \ldots, N
$$

where the following inequalities were used [29]:

$$
\begin{gathered}
z^{i T}(t) B_{i j} z^{j}(t) \leq\left\|B_{i j}\right\|\left\|z^{i}(t) z^{j}\right\|\|(t)\| \\
\left\|z^{i}(t)\right\|\left\|z^{j}(t)\right\| \leq z^{i T}(t) z^{i}(t)+z^{j}(t) z^{j}(t)
\end{gathered}
$$

Consider that the composite system state vector is

$$
z=\left[\begin{array}{llll}
z^{1} & z^{2} & \ldots & z^{N}
\end{array}\right]^{T} \in S=\left(S_{1} \times S_{2} \times \ldots \times S_{N}\right)
$$

Theorem 6. The composite system (45), (51) which satisfies the following condition

$$
\operatorname{Arg}(\operatorname{eig}(\mathcal{M}))>\beta \frac{\pi}{2}
$$

is asymptotically stable, 
where

$$
\mathcal{M}=\left[\begin{array}{ccccc}
\lambda_{\max \left(A_{1}\right)}+\sum_{1}^{N} \gamma_{1 j} & \gamma_{12} & & \ldots & \gamma_{1 N} \\
\gamma_{21} & \lambda_{\max \left(A_{2}\right)}+\sum_{1}^{N} \gamma_{2 j} & & \ldots & \gamma_{2 N} \\
\ldots & \ldots & \ldots & \\
\gamma_{N 1} & \gamma_{N 2} & \ldots & \lambda_{\max \left(A_{N}\right)}+\sum_{1}^{N} \gamma_{N j}
\end{array}\right]
$$

Proof. Consider the following vector Lyapunov function, $\left(V: R^{\sum} n_{i} \rightarrow R^{N}\right)$

$$
(z)=\left[\begin{array}{llll}
V_{1}\left(z^{1}\right) & V_{2}\left(z^{2}\right) & \ldots & V_{N}\left(z^{N}\right)
\end{array}\right]^{T}
$$

where $V_{i}, i=1,2, \ldots, N$ are defined by $(48), V(z)$ is a continuously differentiable vector function and

$$
V\left(z\left(t_{0}\right)\right)=V_{0}
$$

where

$$
\left\|V_{0}\right\|<\delta
$$

and $\delta$ is a positive constant.

The fractional derivation of (14) is

$$
D^{\beta} V(z)=\left[\begin{array}{lllll}
D^{\beta} V_{1}\left(z^{1}\right) & D^{\beta} V_{2}\left(z^{2}\right) & \ldots & D^{\beta} V_{N} & \left(z^{N}\right)
\end{array}\right]^{T}
$$

and according with (48), (50), and Lemma 1 (6), yields

$$
D^{\beta} V_{i}\left(z^{i}(t)\right) \leq 2 z^{i T}(t) D^{\beta}\left(z^{i}(t)\right)=2 z^{i T}(t)\left(A_{i} z^{i}(t)+\sum_{j=1}^{N} B_{i j} z^{j}(t) .\right.
$$

By employing inequality (52) one derives that

$$
\left.D^{\beta} V_{i}\left(z^{i}\right) \leq 2\left(\lambda_{\max \left(A_{i}\right)}+\sum_{i=1}^{N} \gamma_{i j}\right) z^{i T}(t) z^{i}(t)+\sum_{\substack{j=1 \\ j \neq i}}^{N} \gamma_{i j} z^{j T}(t) z^{j}(t)\right)
$$

or,

$$
D^{\beta} V_{i}\left(z^{i}\right) \leq 2\left(\lambda_{\max \left(A_{i}\right)}+\sum_{i=1}^{N} \gamma_{i j}\right) V_{i}\left(z^{i}\right)+\sum_{\substack{j=1 \\ j \neq i}}^{N} \gamma_{i j} V_{j}\left(z^{j}\right)
$$

From (59), it follows that

$$
D^{\beta} V(z(t))=2 \mathcal{M} V(z(t))
$$

If the condition (56) is satisfied, the system (65) is asymptotically stable, such that

$$
\lim _{t \rightarrow \infty} \| V(z(t) \|=0
$$

It can be concluded that

$$
\lim _{t \rightarrow \infty} V_{i}\left(z^{i}(t)\right)=0, i=1,2, \ldots, N
$$

or,

$$
\lim _{t \rightarrow \infty}\left(z^{i}(t)\right)=0, i=1,2, \ldots, N \text { QED }
$$

The control strategy proposed by Theorem 4 based on the Lyapunov vector function (58) allows the use of $(N \times N)$ matrix operators instead of $\left(\sum n_{i} \times \sum n_{i}\right)$ operators as used in [38,39], which considerably simplifies the calculation and implementation effort. 


\subsubsection{Control Systems-Conclusions}

The previous sections discussed the control laws for several classes of models described by fractional-order equations: Fractional Order Linear Systems, Fractional Order Linear Systems with nonlinear components, Time Delay Fractional Order Linear Systems, Time Delay Fractional Order Linear Systems with nonlinear components. The control law was determined for these systems as, $u(t)=-k y(t)$ where the controller gain $k=k_{1}+k_{2}$ and $k_{2}$ verifies the condition $k_{2} \sigma \leq 1$. The component $k_{1}$ satisfies the frequency condition: $\operatorname{Re}(G(j \omega)) \geq-\sqrt{\sigma}, \forall \omega \in(-\infty,+\infty)$, where $G(j \omega)$ is determined by (13), (28), (36), for each class of systems. This inequality can be interpreted as the Nyquist criterion for the critical point $-\sqrt{\sigma}$.

\section{Results}

\subsection{Soft Exoskeleton Glove Control}

An illustrative example of FOLS models is the model of a biomechanical system of a glove exoskeleton used to restore human functions for people with disabilities or who have suffered various traumas. Figure 1 shows the architecture of such a system that represents a complex structure of muscle tissues combined with elements of mechanical structure. An advanced conceptual analysis of the dynamics of these systems indicates the use of fractional calculus in Maxwell's classic stress-strain models [41,42].
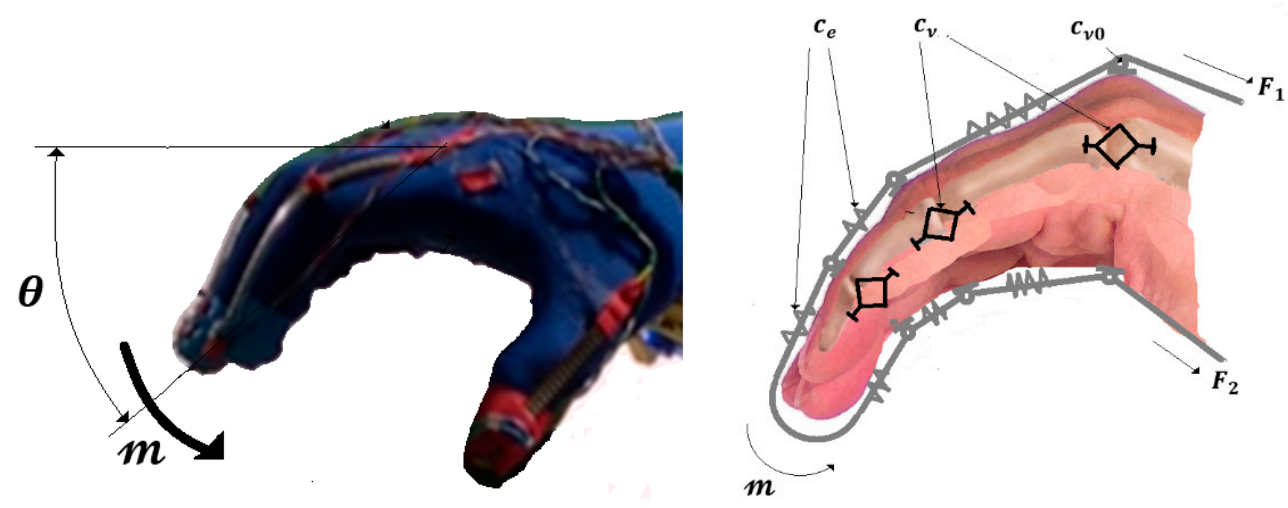

Figure 1. Exoskeleton Glove and its biomechanical components.

\subsubsection{Case 1-FOLS Model}

The dynamic model of this system can be described by the second order transfer function with fractional order component $\beta=1.5$,

$$
Y(s)=\frac{\theta(s)}{m(s)}=\frac{k_{h}}{J s^{2}+c_{v} s^{\beta}+c_{v 0} s+c_{e}}
$$

where $\theta$ is the equivalent angular position of the hand, $m$ is the moment exercised by the driving forces, $J$ is the equivalent inertia moment and $c_{v}, c_{v 0}, c_{e}$ denote coefficients of viscosity and elasticity, respectively, and $k_{h}$ is the gain coefficient. The time model can be written as

$$
J \ddot{\theta}(t)+c_{v} D^{\beta} \theta(t)+c_{e} \theta(t)+c_{v 0} \dot{\theta}(t)=k_{h} m(t)
$$

where the Maxwell's term has the fractional exponent with $\beta=1.5$. The state vector $z=\left[z_{1}, z_{2}, z_{3}, z_{4}\right]^{T}$ is defined as

$$
z_{1}=\theta ; D^{0.5} z_{1}=z_{2} ; D^{0.5} z_{2}=D^{1} z_{1}=z_{3}, D^{0.5} z_{3}=D^{1} z_{1}=z_{4}=\theta^{1.5}, D^{0.5} z_{4}=\ddot{\theta}
$$

The state model can be obtained as

$$
D^{0.5} z(t)=A z(t)+b u(t)
$$


A more complete form also includes a nonlinear term determined by the gravitational components

$$
\begin{gathered}
D^{0.5} z(t)=A z(t)+g(z)+b u(t) \\
z(0)=z_{0}=0
\end{gathered}
$$

where $m$ is the control vector and $A, b, g$ are $(4 \times 4),(4 \times 1),(4 \times 1)$ matrices, respectively,

$$
A=\left[\begin{array}{ccccc}
0 & 1 & 0 & 0 \\
0 & 0 & & 1 & 0 \\
0 & 0 & 0 & 1 \\
-c_{e} / J & 0 & -c_{\nu 0} / J & -c_{v} / J
\end{array}\right] ; b=\left[\begin{array}{c}
0 \\
0 \\
0 \\
k_{h} / J
\end{array}\right] ; c=\left[\begin{array}{l}
c_{1} \\
c_{2} \\
c_{3} \\
c_{4}
\end{array}\right]
$$

The output of the system $y(t)$ is evaluated by direct measuring of the angular position $z_{1}=\theta$ and by estimation of fractional variables $z_{2}, z_{3}$ by using the Grünwald-Letnikov's formula,

$$
y(t)=c^{T} z(t)
$$

The nonlinear component $g(z)$ verifies the inequality (26) with $\mu=2 \times 10^{-3}$. The dynamic system is described by the following parameters [34]: the equivalent inertia is $J=14 \times 10^{-5} \mathrm{~kg} . \mathrm{m}^{2}$, the viscous and elastic coefficients of the joint tissues throughout phalange musculoskeletal system and glove are $c_{v}=0.408 \times 10^{-2} \mathrm{Nm} . \mathrm{s}^{-\mathrm{rad}^{-1}}, c_{e}=0.27 \times 10^{-2} \mathrm{Nm} . \mathrm{rad}^{-1}$, respectively and the damping coefficient is $c_{\nu 0}=0.324 \times 10^{-2} \mathrm{Nm} . \mathrm{s} \cdot \mathrm{rad}^{-1}$.

The matrix $A$ is $1 / 2-$ fractional stable, $\left(\operatorname{Arg}(\operatorname{eig}(A))>\frac{\pi}{4}\right.$, with $\left(\lambda_{1}=-33.48\right.$, $\left.\lambda_{2}=-1.29, \lambda_{3}=0.185+j 0.65, \lambda_{4}=0.185-j 0.65\right)$. According with Theorem 2 , a controller (Figure 2) with $k_{1}=2.4$ is proposed. A matrix $Q=q q^{T}=\operatorname{diag}(0.10 .10 .10 .1]$ is used for Lyapunov Equation (22). The upper matrix bound $\lambda_{\max (P)}=\zeta(Q)=96.8$ is evaluated [29]. The matrix $A^{*}=A-k_{1} b c^{T}+\mu \zeta(Q) \mathrm{I}$ is a Hurwitz matrix $\left(\lambda_{1}=-31.25\right.$, $\left.\lambda_{2}=-2.75, \lambda_{3}=-0.199+j 1.005, \lambda_{4}=-0.199-j 1.005\right)$. The second component is selected as $k_{2}=1.5, \sigma=0.64$ that satisfies the inequality (12). The frequency condition (13) is verified in Figure 3.

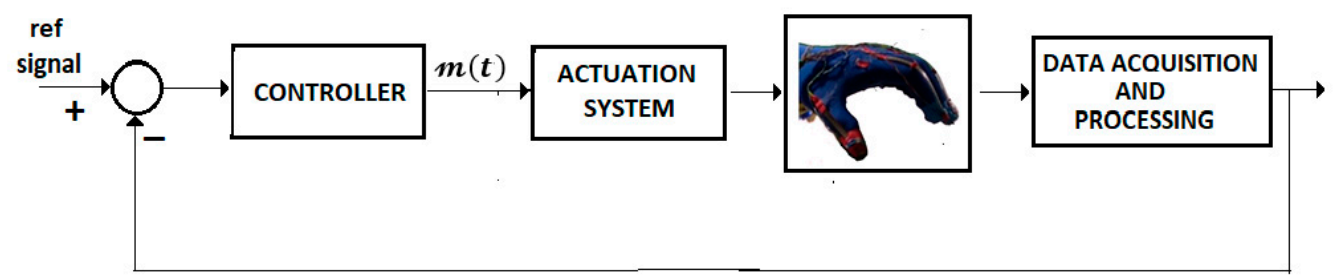

Figure 2. Exoskeleton Glove Control System.

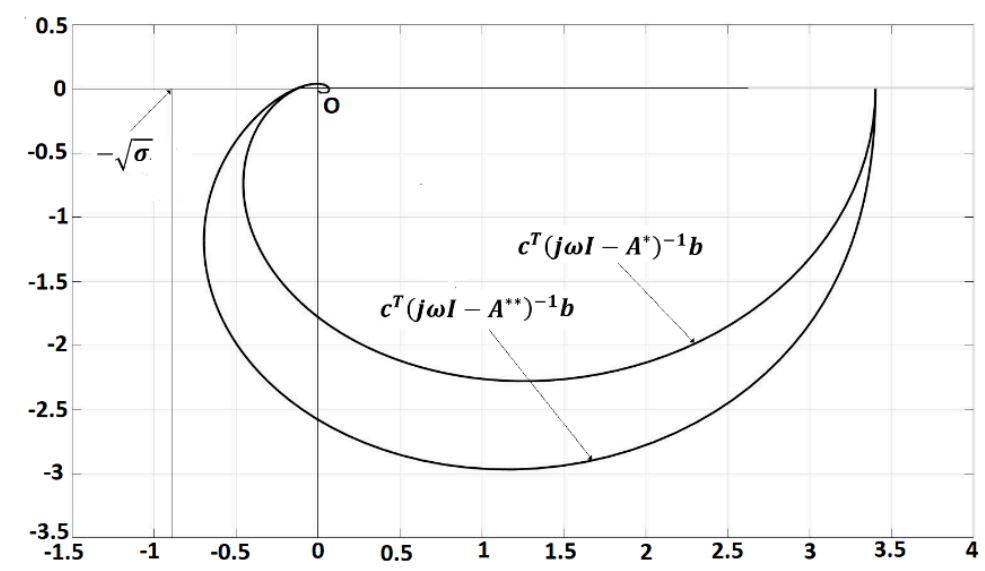

Figure 3. Frequency plots for Exoskeleton Glove model. 
MATLAB/SIMULINK methods are used for the simulation $[4,10]$. The variable trajectories, the physical significance variables as angular position, and the estimated variables by Mittag-Leffler techniques, $z_{2}, z_{3}, z_{4}$ are shown in Figure 4 . The asymptotic stability of all variables, measurable physical significance, and estimated virtual variables is well highlighted. Note the evolution of the angular variable $\theta$, the variable that defines the main coordinate of movement, that satisfies the parameters: the settling time $T_{s}$ and the peak time $T_{p}$ verifies the condition $T_{p} / T_{s}<0.5$ for $T_{s}=5 \mathrm{~s}$ with an overshoot $\delta<0.05 \%$ [40].

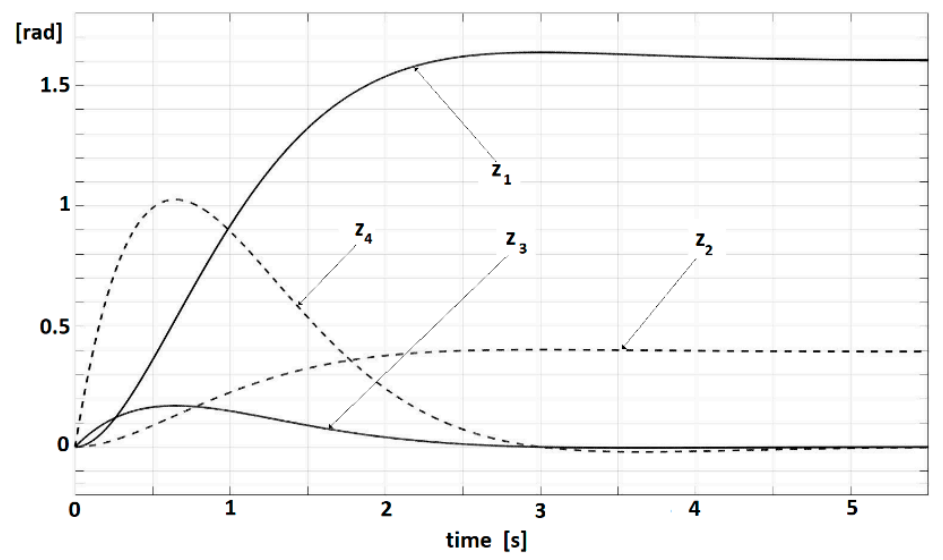

Figure 4. Motion trajectories for FOLS model.

\subsubsection{Case 2-TDFOLS Model}

The reaction time of the man with disabilities, determined by the propagation time in the nervous system as well as the time constants of the muscular system, introduces a delay component $\tau$. Through this component, the dynamic model (69) becomes

$$
Y_{D}(\mathrm{~s})=Y(s) e^{-\tau s}
$$

It is very difficult to evaluate this time delay. Experimental results [42] allow us to identify a domain $\Im=[0.1 ; 0.5] s, \tau \in \Im$. In time domain, the transfer function, becomes

$$
\begin{gathered}
D^{\beta} z(t)=A z(t)+A_{1} z(t-\tau)+b u(t), \mathrm{t} \in[0, \mathrm{~T}], \tau \in \Im \\
\mathrm{z}(t)=0, t \in[-\tau, 0] \\
y(t)=c^{T} z(t),
\end{gathered}
$$

where $A_{1} z(t-\tau)$ is determined by the human decision

$$
m(t-\tau)=k_{h} b_{h} c_{h}^{T} z(t-\tau)
$$

and the matrix coefficients are defined as in (74). According with Theorem 3, a controller with $k_{1}^{*}=7.4$ is used. The matrix $A^{* *}=A-k_{1}^{*} b c^{T}+\mu \zeta(Q)$ I is Hurwitz stable $\left(\lambda_{1}=-23.69, \lambda_{2}=-1.4, \lambda_{3}=-0.35, \lambda_{4}=-0.56\right)$. The gain $k_{2}$ is selected as $k_{2}=3.5$ to satisfy the constraint (37) for $\sigma=0.25$. The factor $\rho$ is evaluated, $\rho=3.2$, and the parameter $\rho^{*}$ is estimated as $\rho^{*}=1.6$ for $\tau \in[0.1,0.5] \mathrm{s}$. The frequency condition (38) is verified (Figure 3 ) and the condition (39) is satisfied. The system trajectories are shown in Figure 5. As in the previous example, we remark the asymptotic stability of all variables, measurable physical significance, and estimated virtual variables. Regarding the angular variable $\theta$, the transient response satisfies the parameters: $T_{p} / T_{s}<0.5$ for $T_{s}=6 \mathrm{~s}$ with an overshoot $\delta<0.08 \%$. 


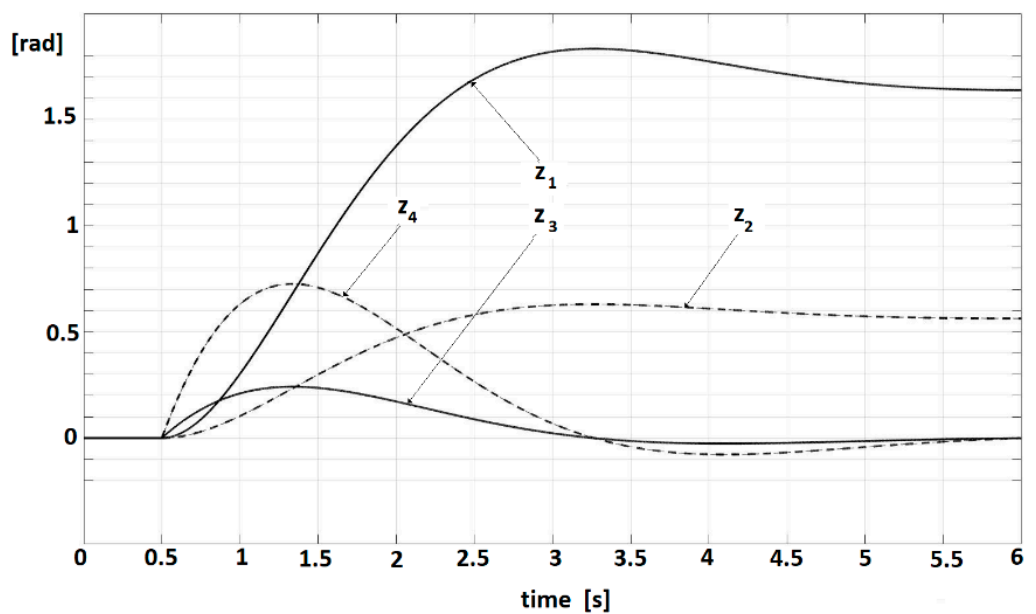

Figure 5. Motion trajectories for TDFOLS model $(\tau=0.5 \mathrm{~s})$.

\subsection{Disabled Man-Wheelchair System Control}

One of the most interesting control applications of some systems defined by fractional order models is the one referring to the control exercised by a disabled person, with health problems regarding locomotor disabilities or cerebral deficiency. Such an example may occur in a person with Parkinson's who drives a wheelchair (Figure 6). This system can be considered as a composite system with two sub-systems: the human operator and the wheelchair.

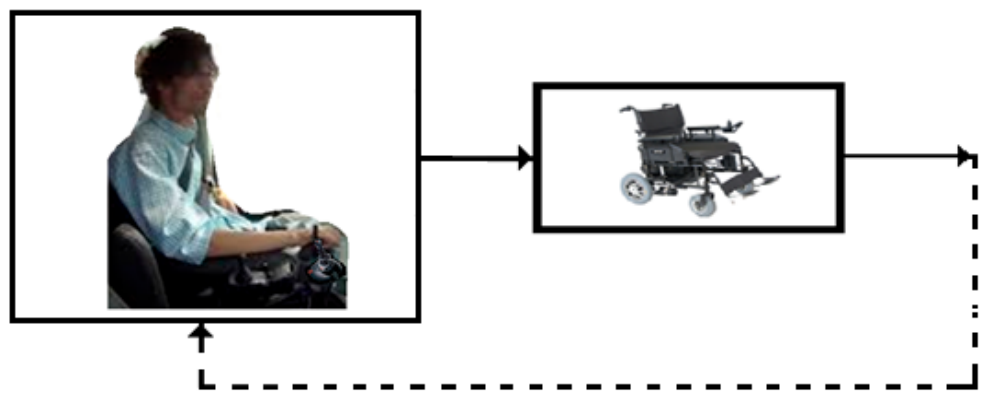

Visual Feedback

Figure 6. Wheelchair control for a person with health problems.

The existence of a handicap of the human operator determines a wheelchair driving with very low performances. For this reason, it is necessary to introduce a controller to improve the control of the movement on the imposed trajectories, (Figure 7). The dynamics of human operator are better characterized by fractional models that more correctly express the control strategy based on memorizing some observations from the history of evolution. The fractional order model is [43-45],

$$
Y_{h}(\mathrm{~s})=\frac{x_{h}(s)}{u_{h}(s)}=\frac{k_{h}}{\left(\tau_{h 1} s^{0.5}+1\right)\left(\tau_{h 2} s+1\right)}
$$

where $x_{h}$ is the human state, $\tau_{h 1}, \tau_{h 2}$ are human time constants, $k_{h}$ is the human gain (see Appendix A), $u_{h}$ defines the human control toward the target, the desired evolution expressed by a desired wheelchair orientation. 


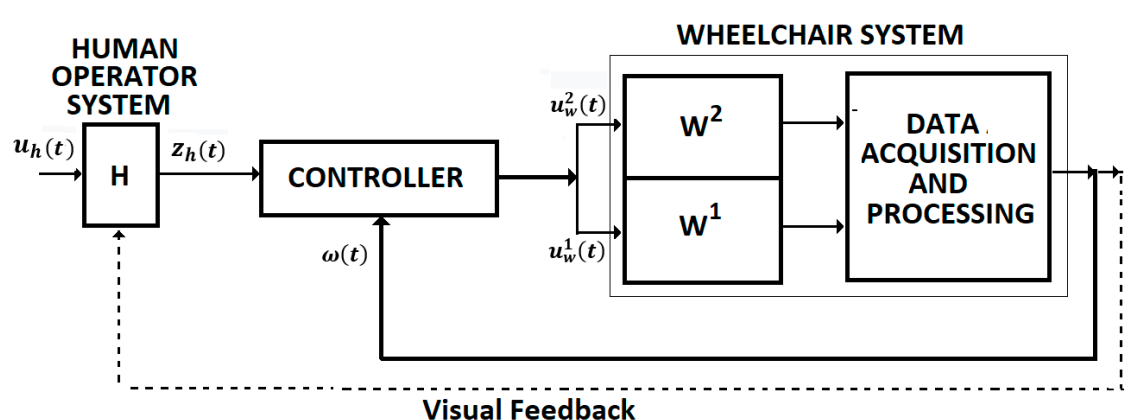

Figure 7. Wheelchair control system.

Rewritten (81) in time, yields

$$
\begin{gathered}
D^{0.5} x_{h 1}(t)=x_{h 2}(t) \\
D^{0.5} x_{h 2}(t)=x_{h 3}(t) \\
D^{0.5} x_{h 3}(t)=-\frac{1}{\tau_{h 1}} x_{h 3}(t)-\frac{1}{\tau_{h 2}} x_{h 2}(t)-\frac{1}{\tau_{h 1} \tau_{h 2}} x_{h 1}(t)+\frac{k_{h}}{\tau_{h 1} \tau_{h 2}} u_{h}(t)
\end{gathered}
$$

or

$$
\begin{gathered}
D^{0.5} z_{h}(t)=A_{h} z_{h}(t)+b_{h} u_{h}(t) \\
y_{h}(t)=c_{h}^{T} z_{h}(t)
\end{gathered}
$$

where $z_{h}=\left[x_{h 1}, x_{h 2}, x_{h 3}\right]^{T}, c_{h}=[0,0,1]^{T}$. The human system is $1 / 2$-fractional order stable with $\lambda_{h 1}=-0.0207, \lambda_{h 2}=-0.0397+0.2163 i, \lambda_{h 3}=-0.0397-0.2163 i$. The human behavior is defined by the derivative component [45],

$$
x_{h 3}(t)=\dot{x}_{h}(t)=\sigma_{h}^{T} z_{w}(t)
$$

where $\sigma^{h}$, a $(4 \times 1)$ matrix, designates the influence of the components of $z_{w}$ on human behavior. The human control $u_{h}$ acts through a negative feedback determined by the information received from the driven system (the wheelchair),

$$
u_{h}(t)=-k_{h}^{*} c_{h w}^{T} z_{w}(t)
$$

where $c_{h w}$ is $(4 \times 1)$ interconnection matrix between the two systems (Appendix A).

The model of the wheelchair is a classical wheelchair model [46] with the control of direction done by the control of the main wheel velocities and detremined by the joystick, The model represents a decoupled drive system, with symmetrical, electrical, and mechanical structure. This symmetry allows to use the following mathematical model

$$
\begin{gathered}
\dot{x}_{w}^{j}(t)=A_{v} x_{w}^{j}(t)+b_{w} u_{w}^{j}(t), j=1,2 \\
A_{w}=\left[\begin{array}{cc}
-v / J & k_{t} / n J \\
-k_{e} / L & -R / L
\end{array}\right], b_{w}=\left[\begin{array}{c}
0 \\
1 / L
\end{array}\right], j=1,2
\end{gathered}
$$

where $x_{w}^{j}=\left[\omega_{j} i_{j}\right]^{T}$ defines the wheelchair state, $u_{w}^{j}$ is the input variable and (index $j=1,2$ defines the motion direction, toward the left and right) and $R, L, J, k_{t}, k_{e}, n, v$ are the electrical and mechanical parameters of the system. The electrical and mechanical parameters are presented in Appendix A.

The fractional order state vector is defined as

$$
z_{w}^{j}=\left[x_{w 1}^{j} x_{w 2}^{j} x_{w 3}^{j} x_{w 4}^{j}\right]^{T}, j=1,2
$$


where

$$
\begin{gathered}
x_{w 1}^{j}=\omega_{j} ; D^{0.5} x_{w 1}^{j}=x_{w 2}^{j} ; D^{0.5} x_{w 2}^{j}=k_{t} / n J x_{w 3}^{j}-v / J x_{w 1}^{j} \\
x_{w 3}^{j}=i_{j} ; D^{0.5} x_{w 3}^{j}=x_{w 4}^{j} ; D^{0.5} x_{w 4}^{j}=-R / L x_{w 3}^{j}-k_{e} / L x_{w 1}^{j}+1 / L u_{w}^{j} ;
\end{gathered}
$$

The dynamic model is

$$
\begin{gathered}
D^{0.5} z_{w}(t)=A_{w} z_{w}(t)+b_{w} u_{w}(t) \\
y_{w}(t)=c_{w}^{T} z_{w}(t)
\end{gathered}
$$

where

$$
\begin{aligned}
& A_{w}=\left[\begin{array}{cccc}
0 & 1 & 0 & 0 \\
-v / J & 0 & k_{t} / n J & 0 \\
0 & 0 & 0 & 1 \\
-k_{e} / L & 0 & -R / L & 0
\end{array}\right] ; b_{w}=\left[\begin{array}{c}
0_{3 \times 1} \\
1 / L
\end{array}\right] ; c_{w}=\left[\begin{array}{c}
c_{\omega} \\
0_{3 \times 1}
\end{array}\right] \text {; } \\
& y_{w}(t)=c_{w}^{T} z_{w}(t)
\end{aligned}
$$

The system (90)-(93) is Hurwitz stable $\left(\lambda_{w 1}=-4.3183+2.0636 i, \lambda_{w 2}=-4.3183-\right.$ $\left.2.0636 i, \lambda_{w 3}=-3.3975+2.9886 i, \lambda_{w 4}=-3.3975-2.9886 i\right)$.

The controller implements the control law for the composite system

$$
u_{w}(t)=-k_{w h} d_{w h}^{T} z_{h}(t)-k_{w w} y_{w}(t)
$$

where $d_{w h}$ a $(3 \times 1)$ matrix, designates the human control components in the global control law (Appendix A).

As proposed in Section 2.2.4, we define the Lyapunov function of the composite system

$$
V=\left[V_{h} V_{w}\right]^{T}
$$

where

$$
\begin{gathered}
V_{h}=z_{h}^{T} z_{h} \\
V_{w}=z_{w}^{T} z_{w} \\
D^{0.5} V=\left[D^{0.5} V_{h} D^{0.5} V_{w}\right]^{T}
\end{gathered}
$$

Using a similar technique as developed in the previous sections, from (83)-(86) and (91)-(94), yields,

$$
\begin{gathered}
D^{0.5} V_{h} \leq \lambda_{\max \left(A_{h}\right)} z_{h}^{T} z_{h}-k_{h}^{*} \lambda_{\min \left(Q_{h w}\right)} z_{w}^{T} z_{w} \\
D^{0.5} V_{w} \leq \lambda_{\max \left(A_{w}^{*}\right)} z_{w}^{T} z_{w}+k_{w h} \lambda_{\max \left(b d_{w h}^{T}\right)} z_{h}^{T} z_{h}
\end{gathered}
$$

where $Q_{h w}=\sigma_{h} c_{h w}^{T}, A_{w}^{*}=A_{w}-k_{w} b_{w} c_{w}^{T}+k_{w h} \lambda_{\max \left(b d_{w h}^{T}\right.}$ I. From (65), (99), obtain

$$
\begin{gathered}
D^{0.5} V=\left[\begin{array}{cc}
\lambda_{\max \left(A_{h}\right)} & -k_{h}^{*} \lambda_{\min \left(Q_{h w}\right)} \\
k_{w h} \lambda_{\max \left(b d_{w h}^{T}\right)} & \lambda_{\max \left(A_{w w}^{*}\right)}^{T}
\end{array}\right] V \\
D^{0.5} V=2 \mathcal{M} V
\end{gathered}
$$

The composite system is $1 / 2$-fractional order stable: $\lambda_{\mathcal{M} 1}=0.23+i 0.45$, $\lambda_{\mathcal{M} 2}=0.23-i 0.45$ with

$$
\operatorname{Arg}(\operatorname{eig}(\mathcal{M}))>\frac{\pi}{4}
$$

The system trajectories, human operator, and wheelchair, for a desired target defined by $\omega_{d}=\pi / 2$ rad are shown in Figure 8 . The good quality of motion can be easily remarked. We remark the asymptotic stability of all variables, measurable physical significance, $\omega_{i}$ and 
estimated virtual variables, $D^{0.5} \omega, D^{0.5} i$ The same behavior is noticed in the parameters that define the human operator, $x_{h 1}, x_{h 2}, x_{h 3}$. Regarding the control angular variable $\omega$, the transient response has an overshoot $\delta<0.01 \%$.

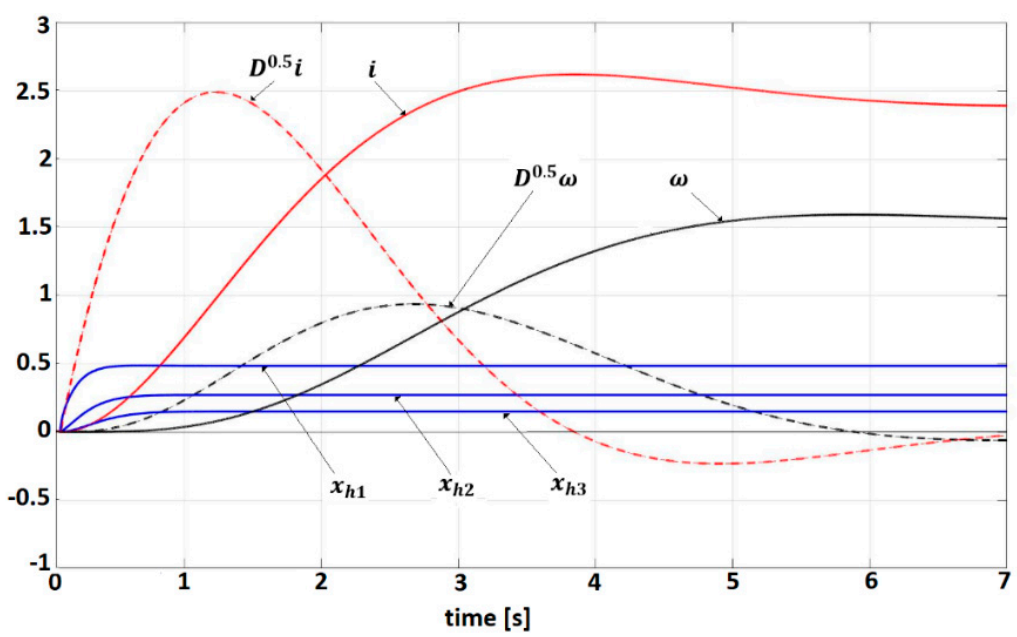

Figure 8. Man-wheelchair system trajectories.

\section{Discussion}

The control techniques presented by the literature are based on algebraic criteria, matrix inequalities and frequency criteria. A selection of these criteria are presented in Table 1. Our paper presents a unitary control method for several classes of FOS systems: Fractional Order Linear Systems, Fractional Order Linear Systems with nonlinear components, Time Delay Fractional Order Linear Systems, Time Delay Fractional Order Linear Systems with nonlinear components. The control laws for these systems have the general form $u(t)=-k y(t)$, where the controller gain satisfies the frequency condition $\operatorname{Re}(G(j \omega)) \geq-\sqrt{\sigma}, \forall \omega \in(-\infty,+\infty)$, where $G(j \omega)$ characterizes the dynamic model of each system.

Table 1. Control Techniques.

\begin{tabular}{ccc}
\hline Papers & Systems & Control Techniques \\
\hline $1,2,4,8,13,20,21$ & Linear FOS & Algebraic criteria \\
\hline $3,5,6,9,12,16,17,18,19,21$ & Nonlinear FOS & $\begin{array}{c}\text { Algebraic criteria } \\
\text { Matrix Inequalities }\end{array}$ \\
\hline $32,33,34$ & Linear FOS & Frequency criteria \\
\hline $11,22,24,25,26,27,28,30$ & Delay FOS & Algebraic criteria \\
\hline
\end{tabular}

\section{Conclusions}

In this paper, Lyapunov techniques are used to formulate frequency criteria for several classes of fractional order systems. Asymptotic stability for the closed loop systems is studied and frequency and algebraic conditions are inferred. FOS linear models, or with nonlinear components are analyzed. A class of delay time FOS systems is analyzed by using an approximate model obtained by the Grunwald-Letnikov formula. Algebraic stability criterion is proposed for systems composed of linear fractional order subsystems. The obtained results were applied on two important applications: the control of a Soft Exoskeleton Glove and the control of a wheelchair of a disabled person.

Author Contributions: Conceptualization, M.I.; methodology, I.D.; software, D.P.; validation, N.P. and M.I.; formal analysis, D.P.; investigation, M.I.; resources, M.I.; data curation, D.P.; writingoriginal draft preparation, N.P.; writing — review and editing, I.D.; visualization, N.P.; supervision, 
I.D.; project administration, N.P.; funding acquisition, M.I. All authors have read and agreed to the published version of the manuscript.

Funding: This research was funded by ROMANIAN SOCIETY OF ROBOTICS, grant number TS137/2019.

Institutional Review Board Statement: Not applicable.

Informed Consent Statement: Not applicable.

Data Availability Statement: Not applicable.

Conflicts of Interest: The authors declare no conflict of interest.

\section{Appendix A}

Appendix A.1. Disabled Human Parameters

- Human time constants: $\tau_{h 1}=0.015 \mathrm{~s}, \tau_{h 2}=0.08 \mathrm{~s}$.

- Human gain $k_{h}=0.78$.

- The matrix $\sigma_{h}$ that designates the influence of the whelchair motion on human behavior,

$$
\sigma_{h}=\left[\begin{array}{llll}
1 & 0 & 0 & 0
\end{array}\right]^{T}
$$

- The interconnection matrix between the human system and wheelchair system,

$$
c_{h w}=\left[\begin{array}{llll}
c_{h w}^{*} & 0 & 0 & 0
\end{array}\right]^{T}
$$

- The matrix of human control components in the global control law,

$$
d_{w h}=\left[\begin{array}{lll}
1 & 1 & 1
\end{array}\right]^{T}
$$

Appendix A.2. Wheelchair System Parameters

Table A1. Wheelchair electrical and mechanical parameters.

\begin{tabular}{ccc}
\hline Parameter & Value \\
\hline $\mathrm{J}$ & $\begin{array}{c}\text { Drive System } \\
\text { Inertia }\end{array}$ & $0.270 \mathrm{~kg} \cdot \mathrm{m}^{2}$ \\
\hline $\mathrm{R}_{\mathrm{a}}$ & $\begin{array}{c}\text { Armature } \\
\text { resistance }\end{array}$ & $0.2957 \Omega$ \\
\hline $\mathrm{L}_{\mathrm{a}}$ & $\begin{array}{c}\text { Armature } \\
\text { inductance }\end{array}$ & $0.082 \mathrm{mH}$ \\
\hline$v$ & Viscous friction coefficient & $0.1044 \mathrm{Nm} \mathrm{s} / \mathrm{rad}$ \\
\hline $\mathrm{k}_{\mathrm{e}}$ & Speed constant & $1.685 \mathrm{rad} / \mathrm{s} / \mathrm{V}$ \\
\hline $\mathrm{k}_{\mathrm{t}}$ & Torque constant & $1.4882 \mathrm{Nm} / \mathrm{A}$ \\
\hline $\mathrm{m}$ & Wheelchair mass & $98 \mathrm{~kg}$ \\
\hline
\end{tabular}

\section{References}

1. Azar, A.T.; Radwan, A.G.; Vaidyanathan, S. (Eds.) Fractional Order Systems: Optimization, Control, Circuit Realization and Applications; Elsevier Inc.: London, UK, 2018; ISBN 978-0-12-816152-4.

2. Makhlouf, A.B. (Ed.) Fractional-Order Systems: Control Theory and Applications, Special Issue, Mathematical Problems in Engineering; Hindawi XML Corpus: London, UK, 2021.

3. Monje, C.; Chen, Y.Q.; Vinagre, D.; Hue, V.; Feliu, V. Fractional-Order Systems and Controls; Springer: London, UK, 2010.

4. Petras, I. Fractional-Order Nonlinear Systems, Modeling, Analysis and Simulation; Higher Education Press: Beijing, China; Springer: Berlin, Germany, 2011.

5. Aguila-Camacho, N.; Duarte-Mermoud, M.; Callegos, J. Lyapunov functions for fractional order systems. Commun. Nonlinear Sci. Numer. Simul. 2014, 19, 2951-2957. [CrossRef] 
6. Argarwal, R.; Hristova, S.; O'Regan, D. Lyapunov functions and strict stability of Caputo fractional differential equations. Adv. Differ. Equ. 2015, 2015, 346. [CrossRef]

7. Agarwal, R.; Hristova, S.; O’Reagan, D. Remarks on Lyapunov functions to Caputo fractional neural networks. Ann. Acad. Rom. Sci. 2018, 10, 169-176.

8. Al-Saggaf, U.M.; Mehedi, I.M.; Mansouri, R.; Bettayeb, M. Rotary flexible joint control by fractional order controllers. Int. J. Control. Autom. Syst. 2017, 15, 2561-2569. [CrossRef]

9. Dadras, S.; Malek, H.; Chen, Y. A Note on the Lyapunov Stability of Fractional Order Nonlinear Systems. In Proceedings of the 13th ASME/IEEE International Conference on Mechatronic and Embedded Systems and Applications, Cleveland, OH, USA, 6-9 August 2017; pp. 123-129.

10. Diethelm, K. The Analysis of Fractional Differential Equations; Springer: London, UK, 2004.

11. Wang, Z.; Wang, C.; Ding, L.; Wang, Z.; Liang, S. Parameter identification of fractional-order time delay system based on Legendre wavelet. Mech. Syst.Signal Process. 2021, 163, 108141. [CrossRef]

12. Li, Y.; Chen, Q.; Podlubny, I. Mittag-Leffler stability of fractional order nonlinear dynamic systems. Automatica 2009, 45, 1965-1969. [CrossRef]

13. Zhao, Y.; Wang, Y.; Liu, Z. Lyapunov Function Method for Linear Fractional Order Systems. In Proceedings of the 2015 34th Chinese Control Conference (CCC), Hangzhou, China, 28-30 July 2015; pp. 1457-1461.

14. Xu, Q.; Zhuang, S.; Xu, X.; Che, C.; Xia, Y. Stabilization of a class of fractional order nonautonomous systems using quadratic Lyapunov functions. Adv. Differ. Equ. 2018, 2018, 14. [CrossRef]

15. Rivero, M.; Rogosin, S.; Machado, J.T. Stability of Fractional Fractional Order Systems. Math. Probl. Eng. 2013, $2013,356235$. [CrossRef]

16. Sabatier, J.; Moze, M.; Farges, C. LMI stability conditions for fractional order systems. Comput. Math. Appl. 2010, 59, 1594-1609. [CrossRef]

17. Sene, N. Lyapunov Characterization of the Fractional Nonlinear Systems with Exogenous Input. Fractal Fract. $2018,2,17$. [CrossRef]

18. Tuan, H.T.; Trinh, H. Stability of fractional-order nonlinear systems by Lyapunov direct method. IET Control. Theory Appl. 2018, 12, 2417-2422. [CrossRef]

19. Zhou, X.F.; Hu, L.G.; Liu, S.; Jiang, W. Stability criterion for a class of nonlinear fractional differential systems. Appl. Math. Lett. 2014, 28, 25-29. [CrossRef]

20. Mashayekhi, S.; Hussaini, M.Y.; Oates, W. A physical interpretation of fractional viscoelasticity based on the fractal structure of media: Theory and experimental validation. J. Mech. Phys. Solids 2019, 128, 137-150. [CrossRef]

21. Oates, W.S.; Smith, R.C. Nonlinear Optimal Control Techniques for Vibration Attenuation Using Magnetostrictive Actuators. J. Intell. Mater. Syst. Struct. 2008, 19, 193-209. [CrossRef]

22. Vadivoo, B.; Raja, R.; Agarwal, R.; Rajchakit, G. A novel controllability analysis of impulsive fractional linear time invariant systems with state delay and distributed delays in control. Interdiscip. J. Discontinuity Nonlinearity Complex. 2018, 7, 275-290. [CrossRef]

23. Khimani, D.; Patil, M. High Performance Super-twisting Control for State Delay Systems. Int. J. Control. Autom. Syst. 2018, 16, 2063-2073. [CrossRef]

24. Koo, M.-S.; Choi, H.-L. Fast Regulation Control of a Class of Input-delayed Linear Systems with Pre-feedback. Int. J. Control. Autom. Syst. 2018, 16, 141-149. [CrossRef]

25. Lazarević, M.P.; Spasić, A.M. Finite-time stability analysis of fractional order time-delay systems: Gronwall's approach. Math Comput. Model. 2009, 49, 475-481. [CrossRef]

26. Wu, B.; Wang, C.-L.; Hu, Y.-J.; Ma, X.-L. Stability Analysis for Time-delay Systems with Nonlinear Disturbances via New Generalized Integral Inequalities. Int. J. Control. Autom. Syst. 2018, 16, 2772-2780. [CrossRef]

27. Xiao, S.; Xu, L.; Zeng, H.-B.; Teo, K.L. Improved Stability Criteria for Discrete-time Delay Systems via Novel Summation Inequalities. Int. J. Control. Autom. Syst. 2018, 16, 1592-1602. [CrossRef]

28. Zhang, X. Some results of linear fractional order time-delay system. Appl. Math. Comput. 2008, 197, 407-411. [CrossRef]

29. Ulukök, Z.; Türkmen, R. Some Upper Matrix Bounds for the Solution of the Continuous Algebraic Riccati Matrix Equation. J. Appl. Math. 2013, 2013, 792782. [CrossRef]

30. Zhe, Z.; Ushio, T.; Ai, Z.; Jing, Z. Novel stability condition for delayed fractional-order composite systems based on vector Lyapunov function. Nonlinear Dyn. 2019, 99, 1253-1267. [CrossRef]

31. Fukuda, K.; Ushio, T. Decentralized Event-Triggered Control of Composite Systems Using M-Matrices. IEICE Trans. Fundam. Electron. Commun. Comput. Sci. 2018, E101-A, 1156-1161.

32. Mousavi, S.S.; Tavazoei, M.S. Stability Analysis of Fractional Order Systems Described in the Lur'e Structure. arXiv 2015, arXiv:1512.02432v1.

33. Lozynskyy, O.Y.; Kalenyuk, P.I.; Lozynskyy, A.O.; Kasha, L.V. A Frequency criterion for analysis of stability of systems with fractional-order derivatives. Math. Modeling Comput. 2020, 7, 389-399. [CrossRef]

34. Zhou, J. Nyquist-Like Stability Criteria for Fractional-Order Linear Dynamical Systems. Control Theory Eng. 2019, 245-258. [CrossRef] 
35. Tepliakov, A.; Alagoz, B.B.; Yeroglu, C.; Gonzales, E.; Hosseinia, H.; Petlemkov, E.; Ate, A.; Cechs, M. Towards Industrialization of FOPID Controllers: A Survey on Milestones of FractionalOrder Control and Pathways for Future Developments. IEEE Access 2021, 9, 21016-21042. [CrossRef]

36. Mohamed, S.M.; Sayed, W.S.; Said, L.A.; Radwan, A.G. Reconfigurable FPGA Realization of Fractional-Order Chaotic Systems. IEEE Access 2021, 9, 89376-89389. [CrossRef]

37. Pappas, G.; Alimisis, V.; Dimas, C.; Sotiriadis, P. Analogue Realization of a Fully Tunable Fractional-Order PID Controller for a DC Motor. In Proceedings of the 2020 32nd International Conference on Microelectronics (ICM), Aqaba, Jordan, 14-17 December 2020; pp. 486-495. [CrossRef]

38. Volos, C.; Pham, V.-T. Advances in nonlinear dynamics and chaos, Chapter 11-design guidelines for physical implementation of fractional-order integrators and its application in memristive systems. In Mem-Elements for Neuromorphic Circuits with Artificial Intelligence Applications; Academic Press: London, UK, 2021; pp. 225-248.

39. Li, Z.; Ding, J.; Wu, M.; Lin, J. Discrete fractional order PID controller design for nonlinear systems. Int. J. Syst. Sci. 2021, 367-378. [CrossRef]

40. Khalil, H. Nonlinear Systems; Prentice Hall: Hoboken, NJ, USA, 2002.

41. Ivanescu, M.; Popescu, N.; Popescu, D.; Channa, A.; Poboroniuc, M. Exoskeleton Hand Control by Fractional Order Models. Sensors 2019, 19, 4608. [CrossRef]

42. PCCA 150/2016 grant of the Executive Agency for Higher Education, Research Development and Innovation Funding (UEFISCDI)Sci. Report.

43. Huang, J.; Chen, Y.; Li, H.; Shi, X. Fractional Order Modeling of Human Operator Behavior with Second Order Controlled Plant and Experiment Research. IEEE/CAA J. Autom. Sin. 2016, 3, 271-280.

44. Aydin, Y.; Tokatli, O.; Patoglu, V.; Basdogan, C. Stable Physical Human-Robot Interaction Using Fractional Order Admittance Control. IEEE Trans. Haptics 2017, 11, 464-475. [CrossRef] [PubMed]

45. Kang, H.G.; Seong, P.H. Information theoretic to man-machine interface complexity evaluation. IEEE Trams. Sys. Man Cyber. 2001, 321, 163-171. [CrossRef]

46. Wolm, P. Dynamic Stability Control of Front Wheel Drive Wheelchair Using Solid State Accelerometer and Gyroscope. Ph.D. Thesis, University of Canterbury, Christchurch, New Zealand, 2009. 